\title{
Case report: Heart rate variability in a patient with coronavirus disease 2019
}

Prof. Dr. Reiner Buchhorn ${ }^{\mathrm{a}}$, Christoph Baumann ${ }^{\mathrm{b}}$, Christian Willaschek $^{\mathrm{c}}$

${ }^{\text {a} C a r i t a s-K r a n k e n h a u s ~ B a d ~ M e r g e n t h e i m, ~ D e p a r t m e n t ~ o f ~ P e d i a t r i c s, ~ U h l a n d s t r a ß e ~ 7, ~ B a d ~}$

Mergentheim, Germany. This author takes responsibility for all aspects of the reliability and freedom from bias of the data presented and their discussed interpretation.

${ }^{\mathrm{b}}$ University of Wuerzburg, Medical Faculty, Josef-Schneider-Straße 2, Würzburg, Germany. This author takes responsibility for all aspects of the reliability and freedom from bias of the data presented and their discussed interpretation.

${ }^{\mathrm{c} C a r i t a s-K r a n k e n h a u s ~ B a d ~ M e r g e n t h e i m, ~ D e p a r t m e n t ~ o f ~ P e d i a t r i c s, ~ U h l a n d s t r a ß e ~ 7, ~ B a d ~}$ Mergentheim, Germany. This author takes responsibility for all aspects of the reliability and freedom from bias of the data presented and their discussed interpretation.

Corresponding author: Christoph Baumann

E-mail: Christoph.baumann1993@gmail.com

Conflict of Interests: The authors have no conflict of interests to declare. 


\begin{abstract}
This case study investigates the heart rate (HR) and heart rate variability (HRV) in a patient with coronavirus disease 2019 (COVID-19). We report the case of a 58-year old male who contracted COVID-19. During his disease, 24-hour Holter electrocardiography (ECG) was performed continuously. For comparison, his 24-hour Holter ECGs from the previous 10 years were available. In this patient, COVID-19 was associated with a decrease in HR and a paradoxical decline in HRV. An abrupt decline in HRV and a decrease in HR may signal the onset of COVID-19 before common symptoms such as dry cough or fever appear. In addition, HRV and HR measurements may help to evaluate the course of the disease.
\end{abstract}

Keywords: COVID-19 virus disease; Autonomic nervous system; Electrocardiography; Heart rate 


\section{Introduction}

Corona virus disease (COVID-19) has been declared a pandemic by the WHO. The global mortality rate is about 3.4\% (WHO, 2020), and observational studies indicate that preexisting conditions such as obesity, cardiovascular disease, diabetes, chronic respiratory disease, hypertension, and cancer increase this rate. To date, no clear parameters that predict disease severity have been identified. To reduce infection rates, timely self-isolation of infected individuals is necessary. However, because infective virus is shed before symptoms appear, self-isolation may begin too late. Thus, early indicators of COVID-19 are needed.

Earlier diagnosis of COVID-19 may be facilitated by heart rate (HR) and heart rate variability (HRV) monitoring. HR and HRV parameters could not only help to detect COVID-19 in a timely manner but could also help to identify patients at risk for cardiovascular/pulmonary complications. Additionally, HRV and HR parameters may help to assess the course of the disease. HRV measures variations in the normal-to-normal (NN) interval, reflecting a complex interplay of feedback loops, thermogenesis, intrinsic mechanisms of pacemaker cells, and parasympathetic and sympathetic tone. To this day, no study has investigated HRV and HR in COVID-19.

\section{Main text}

In this study, we monitored and analyzed the HRV and HR of a 58-year old pediatric cardiologist who was infected with COVID-19. He had a medical history of atrial fibrillation After pulmonary vein isolation, no cardiac dysrhythmias were detected.

During his disease, 24-hour Holter electrocardiography (ECG) was performed continuously (Pathfinder, Spacelabs Healthcare, Snoqualmie, WA, USA). Additionally, his 24-hour Holter ECGs from the previous 10 years were available. The HR and HRV parameters, including standard deviation of NN intervals (SDNN) and total power (TP), were calculated in the standard manner over 24-hour intervals. The HR and HRV changes are shown in the line 
chart in Figure 1. As a baseline value, the mean HR and mean SDNN were calculated from 24-hour Holter ECGs that were recorded over the past two years (31.10.2018: HR, 73 bpm; SDNN, 118.3 ms; 25.01.2019: HR, 74 bpm; SDNN, 103.9 ms; 30.03.2019: HR, 79.4 bpm; SDNN, $112.6 \mathrm{~ms})$. Additionally, 24-hour ECGs from the past 10 years $(\mathrm{N}=29)$ and 24-hour Holter ECGs recorded during COVID-19 infection were used to plot the relationship between total spectral power (TP) (a measure of overall autonomic activity) and HR (Figure 2).

Our main finding was a decrease in HR during COVID-19 (Figures 1 and 2), with a parallel decline in HRV (Figure 1). The decline in HRV was surprising because HRV parameters typically increase fundamentally with decreasing HRs and increasing IBIs for mathematical and physiological reasons (M. Boyett, Wang, \& D'Souza, 2019; M. R. Boyett, 2017; Gąsior, Sacha, Jeleń, Zieliński, \& Przybylski, 2016; Monfredi et al., 2014; Sacha, 2013). In reviewing 1000 24-hour Holter ECGs from these publications, we only observed this phenomenon of HRV saturation in patients with severe anorexia nervosa and athletes. The observed HRV and HR changes are reminiscent of HRV changes that accompany the aging process (Figure 2) (van den Berg et al., 2018). A possible explanation of HRV saturation is discussed in our preprint (Baumann, 2019). Moreover, the observed HRV and HR changes may be explained by potential neuroinvasion of SARS-CoV-2. On day 16, the HRV and HR increased and approached pre-disease values, which can be interpreted as signs of recovery as symptoms disappeared (Figure 1).

In conclusion, further research is needed to clearly identify HRV and HR patterns in patients with COVID-19. In particular, the role of physical activity levels as a confounder of HRV and HR should be investigated. Nighttime ECG recordings or standardized 5-minute ECG recordings may be less prone to confounders.

User-friendly HR watches, mobile ECG devices, or chest straps may be practical options for use in ambulatory settings to reveal HRV and HR patterns that signal health risks. Thus, the 
development of algorithms and advances in machine learning techniques to recognize parameters indicating severe diseases like COVID 19 are needed. To date, similar algorithms focus on stress regulation. In case presented in this study, a fitness tracker (LifeTrak Zoom HRV, LifeTrak, USA) and the Samsung Health Galaxy wearable App (https://www.samsung.com/de/apps/samsung-health/) incorrectly interpreted the low heart rates as relaxation instead of COVID-19.

Data Availability: The datasets analyzed in this study are available from Reiner Buchhorn or Christoph Baumann upon request.

The authors state that they abide by the authors' responsibilities and ethical publishing guidelines of the International Cardiovascular Forum Journal (Shewan LG, 2018). 


\section{References}

Baumann, C., Willaschek, C., \& Buchhorn, R. (2019). Heart rate regulation in patients with anorexia nervosa. . PsyArXiv. doi:https://doi.org/10.31234/osf.io/qpgz4

Boyett, M., Wang, Y., \& D'Souza, A. (2019). CrossTalk opposing view: Heart rate variability as a measure of cardiac autonomic responsiveness is fundamentally flawed. The Journal of physiology, 597(10), 2599-2601. doi:10.1113/JP277501

Boyett, M. R. (2017). Last Word on Point:Counterpoint. 123(3), 694-694. doi:10.1152/japplphysiol.00542.2017

Gąsior, J. S., Sacha, J., Jeleń, P. J., Zieliński, J., \& Przybylski, J. (2016). Heart Rate and Respiratory Rate Influence on Heart Rate Variability Repeatability: Effects of the Correction for the Prevailing Heart Rate. Frontiers in Physiology, 7, 356-356. doi:10.3389/fphys.2016.00356

Monfredi, O., Lyashkov, A. E., Johnsen, A.-B., Inada, S., Schneider, H., Wang, R., . . Boyett, M. R. (2014). Biophysical characterization of the underappreciated and important relationship between heart rate variability and heart rate. Hypertension, 64(6), 1334-1343. doi:10.1161/HYPERTENSIONAHA.114.03782

Sacha, J. (2013). Why should one normalize heart rate variability with respect to average heart rate. Front Physiol, 4, 306. doi:10.3389/fphys.2013.00306

Sammito, S., \& Bockelmann, I. (2016). Reference values for time- and frequency-domain heart rate variability measures. Heart Rhythm, 13(6), 1309-1316. doi:10.1016/j.hrthm.2016.02.006

Shewan LG, C. A., Henein MY. . (2018). Authors' Responsibilities and Ethical Publishing. . International Cardiovascular Forum Journal; doi: DOI: 10.17987/icfj.v13i0.525

van den Berg, M. E., Rijnbeek, P. R., Niemeijer, M. N., Hofman, A., van Herpen, G., Bots, M. L., . . Kors, J. A. (2018). Normal Values of Corrected Heart-Rate Variability in 
10-Second Electrocardiograms for All Ages. Frontiers in Physiology, 9, 424-424. doi:10.3389/fphys.2018.00424

WHO. (2020). https://www.who.int/dg/speeches/detail/who-director-general-s-openingremarks-at-the-media-briefing-on-covid-19---3-march-2020. 


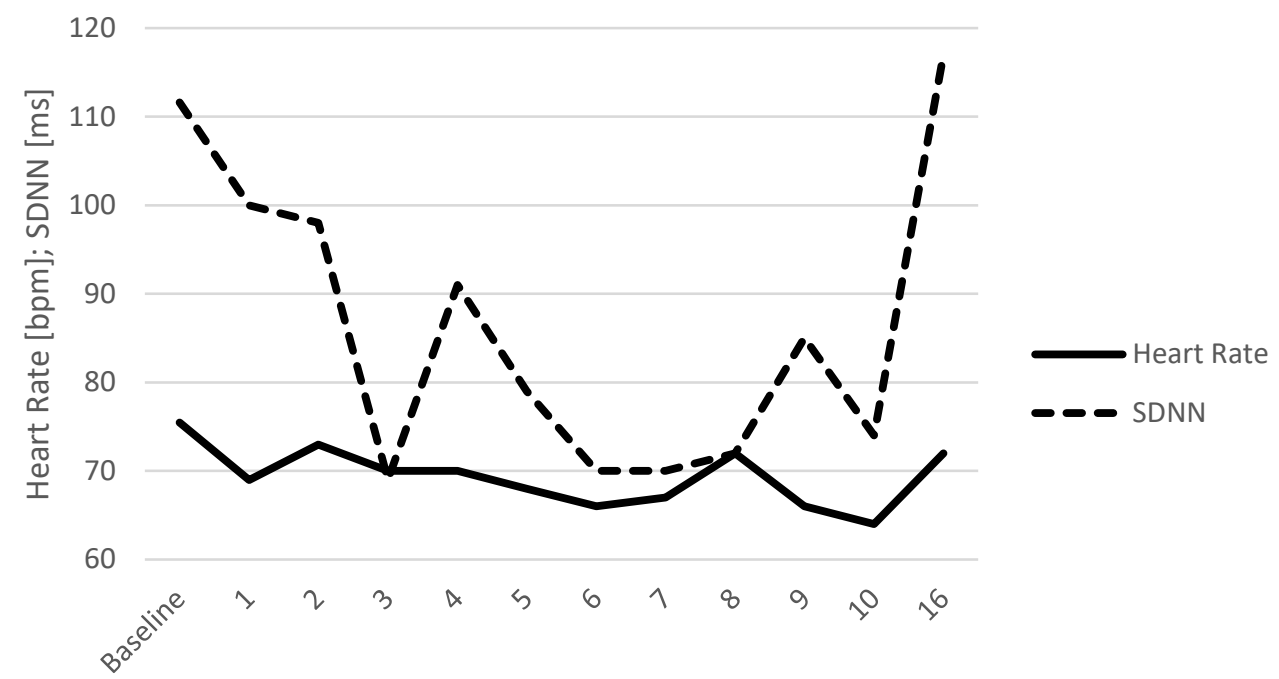

COVID-19 Day

Figure 1. HR and SDNN levels before and during COVID-19. The patient contracted COVID19 on April $5^{\text {th }}$. On April 10 (day 1) sub-febrile temperatures were noticed and limb pain and dysgeusia appeared. On April 23 (day 13) SARS-CoV2 PCR was negative. Throughout the course of the disease, oxygen saturation was high (>98\%). Eventually, the reported clinical symptoms completely disappeared. Notably, the SDNN and HR plots cross during COVID-19 infection. 


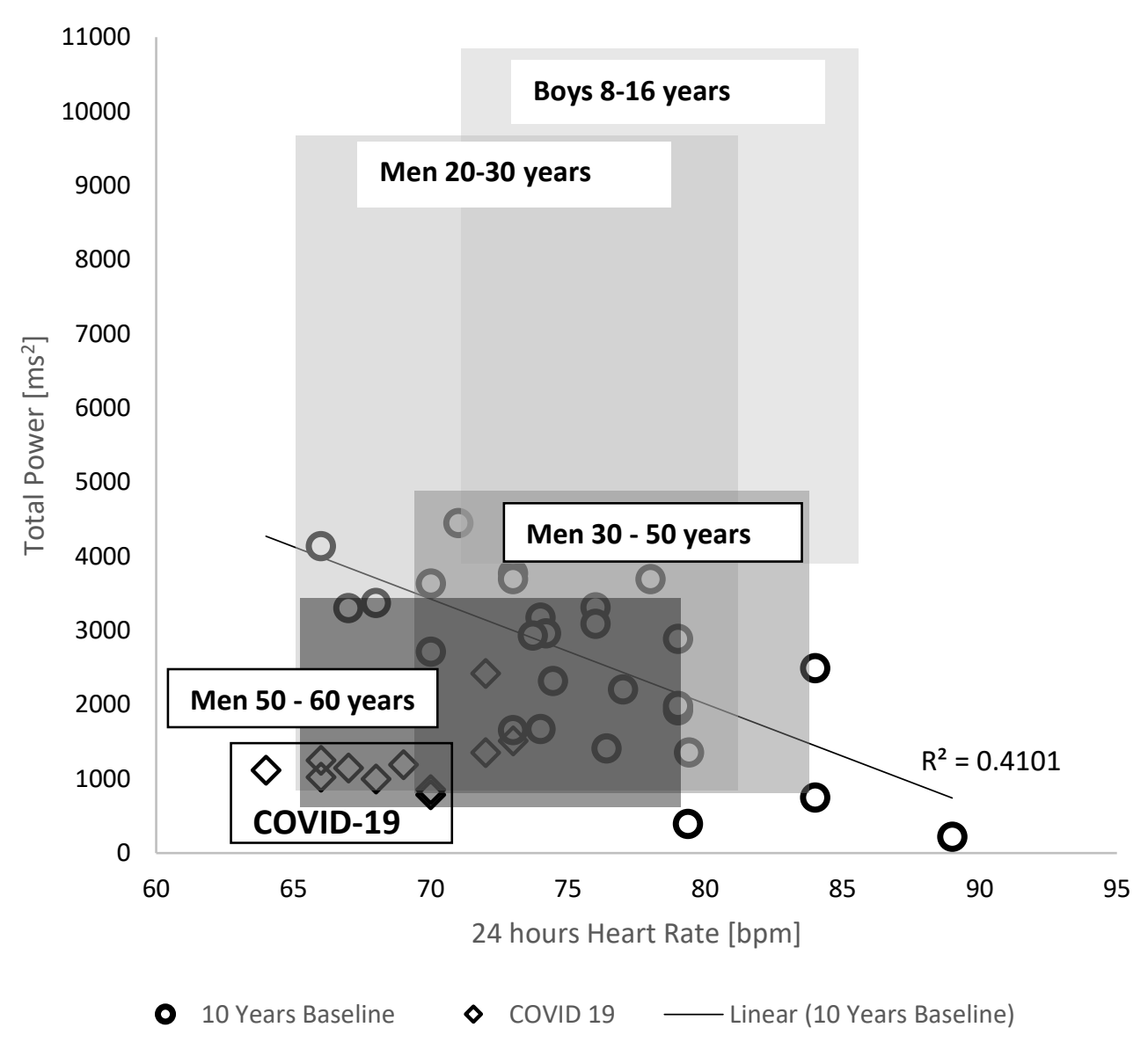

Figure 2. Relationship between TP and HR.

Most of the TP and HR values from 24-hour Holter ECGs of the patient over the past 10 years were within the normal range for his age group (German men, aged 50-60 years; quadrangles represent standard deviations) (Sammito \& Bockelmann, 2016). During COVID-19, his TP dropped to very low values without an increase in the mean HR. The rhombus marking a TP of $2500 \mathrm{~ms}^{2}$ corresponds to day 16 after infection and indicates recovery. 